\title{
Elliptical BandPassThree Dimensional Frequency Selective Surface with Multiple Transmission Zeros
}

\author{
Bimal Raj Dutta ${ }^{1}$, Binod Kumar Kanaujia ${ }^{2}$, Chhaya Dalela ${ }^{3}$ \\ ${ }^{1}$ Department of Electronics \& Communication, A.N.A College of Engineering \&Management Studies, India \\ ${ }^{2}$ School of Computational and Integrative sciences, Jawaharlal Nehru University, India \\ ${ }^{3}$ Department of Electronics \& Communication, JSS Academy of Technical Education, India
}

\begin{abstract}
Article Info
Article history:

Received Jun 3, 2018

Revised Jul 15, 2018

Accepted Aug 27, 2018

\section{Keywords:}

Elliptic Filters

Frequency Selective Surface

Radio wave propagation

Resonators

Transmission line theory

ABSTRACT

An elliptic band pass response three-dimensional Frequency Selective Surface (3D FSS) is designed from a single unit cell of 2D array of two shielded microstrip lines. The designed FSS provides pseudo-elliptic bandpass frequency response $(5.4-9.6) \mathrm{GHz}$ with its application in long-distance radio telecommunications and space communications etc. The four transmission zeros at $5.4 \mathrm{GHz}, 9.6 \mathrm{GHz}, 12.4 \mathrm{GHz}$ and $15 \mathrm{GHz}$ provides wide out-of-band frequency rejection. The 3D FSS is independent of the variations in the incident angle of the plane wave up to 60 degree. Each unit cell is a combination of two shielded microstrip lines with one having an air gap and the other one having in between rectangular metallic plate. When a TE polarized plane wave incidents perpendicular to the perfect electric conductor (PEC) boundary walls shielded microstrip lines, it results in two quasi-TEM modes namely air and substrate mode. The 3D FSS consists of multiple resonators with a multimode cavity having number of propagating modes. These resonating modes in phase provide transmission poles and when out of phase give transmission zeros. The 3D FSS structure is simulated using Ansys HFSS software with improved performance over 2DFSS, for many practical applications such as antenna sub-reflector, radomes and spatial filters.
\end{abstract}

Copyright $(9) 2018$ Institute of Advanced Engineering and Science. All rights reserved.

\section{Corresponding Author:}

Bimal Raj Dutta,

Department of Electronics \& Communication,

A.N.A College of Engineering \&Management Studies, India.

Email: brajdutta@gmail.com

\section{INTRODUCTION}

The electromagnetic waves appear like Frequency selective surface spatial filters with band pass or band stop characteristics. They have been the topic of research for the previous few decades and have been used in many practical applications such as spatial filters, radomes, polarizer, antenna sub-reflectors etc [1-3]. Traditional two-dimensional periodic 2D FSS structures were designed by the periodic array combination of unit cells which were constructed by either etching the slots from the conducting plates or by printing the conducting patches over the dielectric layer [4-5]. These traditional two-dimensional periodic 2D FSS structure's single layer suffers from poor filtering response, poor selectivity, unstable angular response and narrow bandwidth.

With the advancement in the field of antennas and communication it became desirable to realize thin FSS structures to achieve high selectivity and stable frequency response under different angles of incidence. Requirement of such advanced FSS structures lead the research towards a new class of FSS structures called three-dimensional FSS from the traditional 2D FSS [6-7]. The 3D FSS concept gives realization of compact and high performance FSS structures. On cascading number of 2D FSS layers with in between dielectric spacing results to a significant improved filtering response [8-9]. The cascaded 2D FSS does not obtain 
elliptic response; it provides the filtering response that follows Butterworth or Chebyshev function due to the formation of direct-coupled resonators [10]. Practical applications of the FSS structures demands stability in the variations of angular response, where 2D FSS structures showed unstable frequency response for the variations in the incidence angle.

The 3D FSS structures have shown far more stability in the frequency response under variations in the incidence angle. In Comparison to conventional multilayer 2D FSS structures, 3D FSS structures can be designed to obtain broad out-of-band frequency rejection with the multiple transmission zeros.

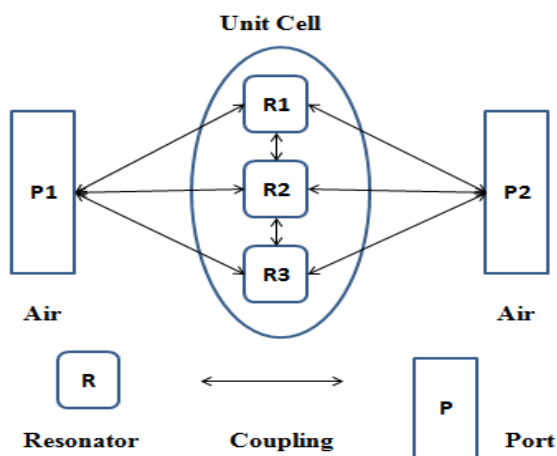

(a)

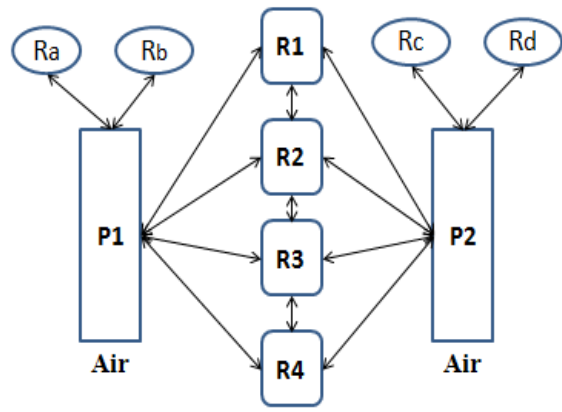

(b)

Figure 1. (a) 3D FSS unit cell showing cross-coupling and coupling between the two ports via multiple resonators to obtain transmission zeros/poles and pseudo-elliptic response, (b). Equivalent model of any 3D FSS with multiple resonators

Elliptic filtering response in 3D FSS is achieved with the increase of the level of cross-coupling between the resonators as shown in Figure 1(a) which were absent in cascaded previous multilayer 2D FSS designs. Elliptic filtering response in 3D FSS design can be achieved with the help of two ports of the unit cell coupled with more than one resonator as shown in Figure 1 (a) [11-12]. The concept of 3D FSS will produce multiple transmission poles/zeros through multimode resonators. Recent research in 3D FSS technology have been around the band-pass or band-reject structures, they are designed with the unit cell of a single shielded microstrip with a short via hole between microstrip line and ground [12-13]. The designed unit cell of three-dimensional FSS was then geometrically modified to add on an extra transmission zero in each modification. Thus maximum three transmission zeros have been obtained for the band-pass 3D FSS structure with pseudo-elliptic response [14-15]. In this design of 3D FSS four transmission zeros with bandpass elliptic response, and broad frequency band rejection has been studied.

Unlike the previous 3D FSS structures, the unit cell of the 3D FSS structure is designed and studied with two shielded microstrips lines placed parallel to each other. Both the microstrip lines are connected to their respective ground through short via and a rectangular metallic plate being placed one over another microstrip line. The designed three-dimensional FSS with a 2D array arrangement of double shield microstrip lines placed perpendicular, to support two quasi-TEM modes namely air and substrate modes [16]. Transmission zeros are introduced at the desired finite frequencies of a pass-band of $3.3 \mathrm{GHz}$ in the $\mathrm{C}$ frequency band (4-8) $\mathrm{GHz}$ and $\mathrm{X}$ frequency band (8-12) $\mathrm{GHz}$ with broad frequency band rejection.This improves the selectivity of the operating bandwidth.

\section{RESEARCH METHOD}

Any 3D FSS structure is represented by an equivalent model of Figure 1(b) which has an equivalent model of total eight resonators. Here four resonators $R_{1}, R_{2}, R_{3}$ and $R_{4}$ resonators are used to connect input port and output port, resonators $R_{a}$ and $R_{b}$ are connected to the input port only and resonators $R_{c}$ and $R_{d}$ are connected to the output port only. Every Resonator defines their propagating mode. $R_{1}, R_{2}, R_{3}$ and $\mathrm{R}_{4}$ resonators will provide transmission zeros/poles at their respective resonant frequencies. The additional transmission zeros will be provided by resonators $R_{a}, R_{b}, R_{c}$ and $R_{d}$ at the desired frequencies. These additional transmission zeros increases operating band width of a band-stop FSS, which improves the selectivity of a band-pass FSS. The production of the desired number of transmission zeros/poles at finite frequencies can be resulted by controlling the number of resonators and their resonances that makes 
transmission zeros 3D FSS structures more advantageous than 2D FSS structures [14]. 2D FSS can be considered a special case of this equivalent model of three-dimensional FSS with one resonator or mode that results in a single transmission zero/pole which provides poor filtering performance.

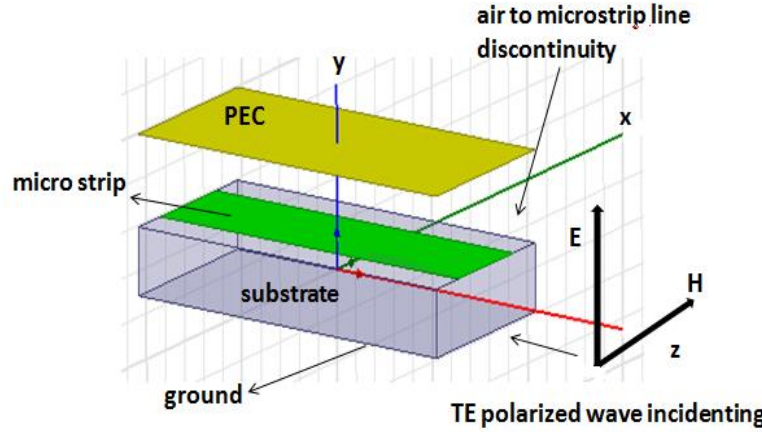

Figure 2(a). Basic unit cell of a 3D FSS

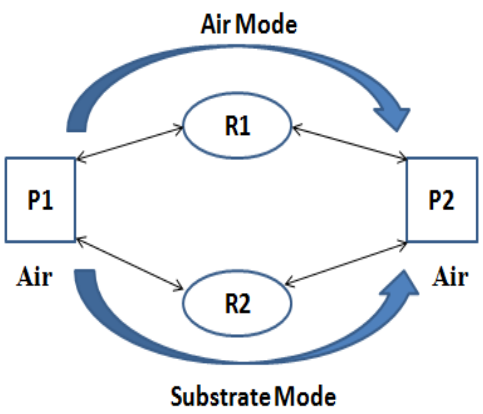

Figure 2(b). Propagating modes of a basic unit cell of a 3D FSS

The working principle of 3D FSS can be reviewed by showing that how the resonators are formed. As shown in Figure 2(a) we have a basic unit cell that is used as a building block for three-dimensional FSS structures.

Here a plane wave with TE polarization (electric field) oriented perpendicular to the shielded microstrip patch incidents at the discontinuity of air-to-microstrip line, two propagating modes namely air and substrate modes are created. These modes link the input and output ports [6]. Due to the wave propagation within two guided media (air and substrate), microstrip line supports quasi-TEM waves instead of pure TEM wave [16]. In quasi-TEM modes the longitudinal waves of the field for the air and substrate modes of a microstrip line exist and these are much smaller than the transverse waves [17].

$$
\begin{aligned}
& \mathrm{E}_{\mathrm{l}} \neq 0, \mathrm{H}_{\mathrm{l}} \neq 0 \\
& \left|\mathrm{E}_{\mathrm{l}}\right| \ll\left|\mathrm{E}_{\mathrm{t}}\right|,\left|\mathrm{H}_{\mathrm{l}}\right| \ll\left|\mathrm{H}_{\mathrm{t}}\right|
\end{aligned}
$$

The signals are travelling through the air path from $\mathrm{p} 1$ port to the other $\mathrm{p} 2$ port at low frequencies. They provide low-pass response, as the frequency of operation increases these signal travel through two paths (air and substrate). The substrate path resonator will always resonate first than the air path resonator since the guided wavelength $\left(\lambda_{\mathrm{g}}\right)$ of the substrate path is less than the air path $\left(\lambda_{0}\right)$ [14, 17]. In Figure 2(b) of air and substrate propagating modes of the basic unit cell, the two possibilities are resulted (a) the generation of transmission poles when the electrical length of a resonator becomes equal to $\pi$, (b) the generation of transmission zeros due to the phase difference of 1800 between the signals in the air and substrate path. Thus for a 3D FSS basic unit cell structure as shown in Figure 3, we obtain two transmission poles and a single transmission zero. By introducing a short via hole between the microstrip line and the ground in the designed unit cell of Figure 3(a) an increment in number of resonator (three resonators) was observed [14]. Further a rectangular metallic plate above the microstrip line in air medium was inducted in the previous design resulted in more number of resonators (four resonators) as shown in Figure 3(b). This increment in the number of resonators resulted in the increment of number of transmission zeros. With the addition of $\mathrm{T}$ shape metallic plate along with small air gap above the microstrip line as discussed in [14], maximum three transmission zeros were achieved, one below the pass-band and two above the pass-band. Thus the geometrical modifications in the basic unit cell resulted in 3D FSS structure that showed improvement in the selectivity, filter response (pseudo-elliptic response) and stable angular response. 


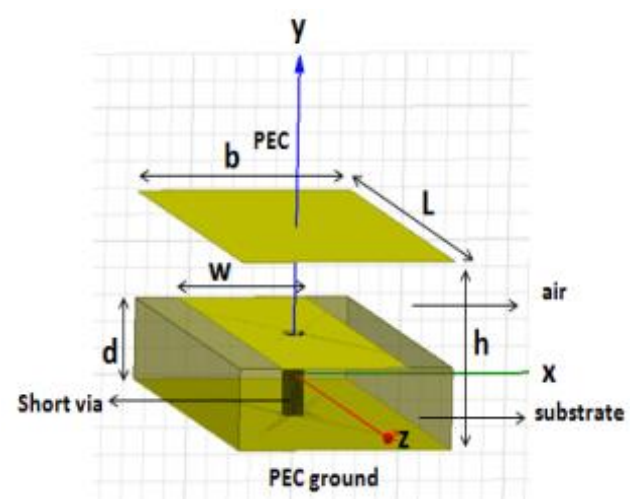

Figure 3(a). Unit cell of a 3D FSS structure structure with short via hole

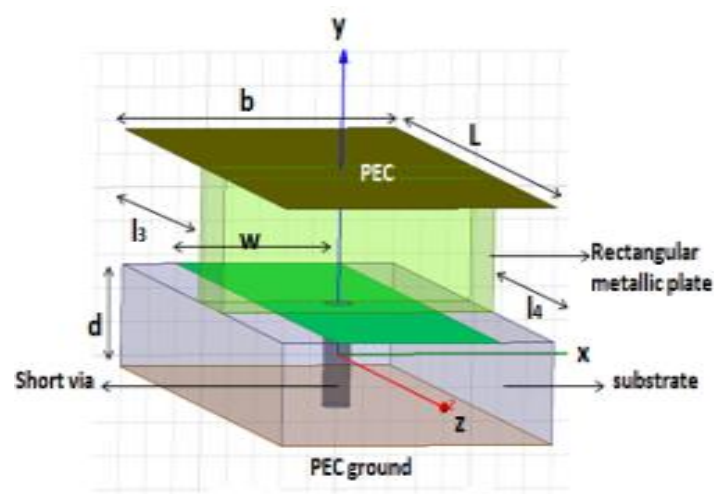

Figure 3(b). Unit cell of a 3D FSS structure with short via hole with only short via hole and Rectangular metallic plate

\subsection{Structure Description}

A 3D view of a three dimensional band-pass FSS is shown in Figure 4(a) and Figure 4(b). Unlike the traditional 3D FSS structures the unit cell of the 3D FSS designed in this paper consists of dual microstrip line placed parallel to each other, each placed over their respective substrate. The unit cells shown above in Figure 3(a), (b) and discussed in [14] have been used in the designing of the unit cell for the band-pass 3D FSS structure discussed in this paper. The designed 3D FSS structure is a 2D periodic array combination of dual shielded microstrip lines placed in $\mathrm{z}$ direction vertically.

The array of Figure 4 is a combination of $4 \times 4$ unit cells. Here the unit cells of Figure 3 are placed one above the other to design the unit cell of Figure 3 . The unit cell length in the $\mathrm{y}$ direction is $2 * \mathrm{~h}=$ $7.048 \mathrm{~mm}$, which is a combination of two substrate regions with microstrip line placed over them, one air region and the other air region with a rectangular metallic plate. The length of each substrate in y direction is $\mathrm{d}=1.524 \mathrm{~mm}$. Unit cell $/$ microstrip line length in $\mathrm{z}$ direction is $\mathrm{L}=10.5 \mathrm{~mm}$. Unit cell $/$ substrate length in $\mathrm{x}$ direction is $\mathrm{b}=5 \mathrm{~mm}$. The substrate material used in this design is Rogers RO3003 with a dielectric constant 3 .

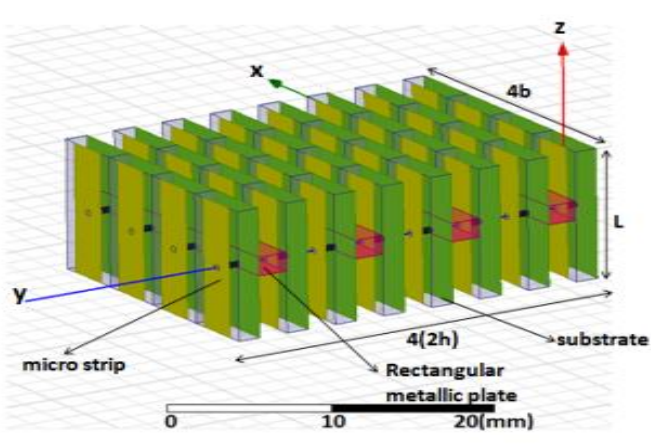

(a)

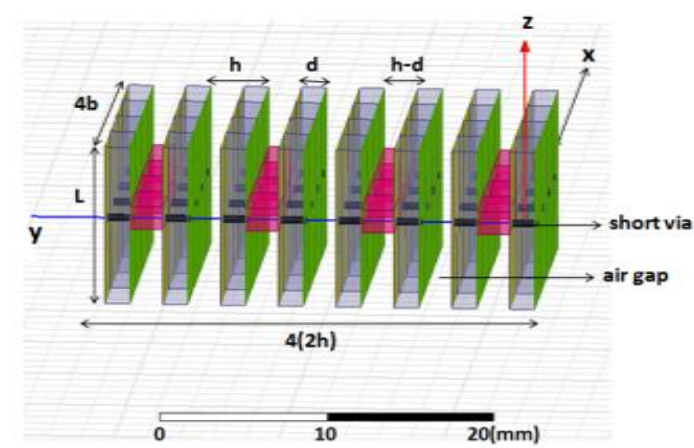

(b)

Figure 4(a)-(b). 3D view of the Three-dimensional band-pass FSS structure

The microstrip line width in $\mathrm{x}$ direction is $\mathrm{w}=3 \mathrm{~mm}$. Two shorting via of diameter $\mathrm{D}=0.5 \mathrm{~mm}$ are also included in the unit cell to connect the centre of both the microstrip lines to their respective grounds. The unit cell reappear after a distance of " $b$ " in $x$ direction and " $2 * h$ " in $y$ direction in this formation of three dimensional FSS array. The rectangular metallic plate is placed in a way to define the distance between the metallic plate and the two ends of the microstrip line. They are $13=5 \mathrm{~mm}$ and $14=4 \mathrm{~mm}$. Each unit cell is having a PEC boundary wall in $\mathrm{x}-\mathrm{z}$ plane. The TE polarized plane wave in $\mathrm{z}$ direction will be incident at incoming port 1 (air to microstrip line discontinuity) as per Figure 3(b). 


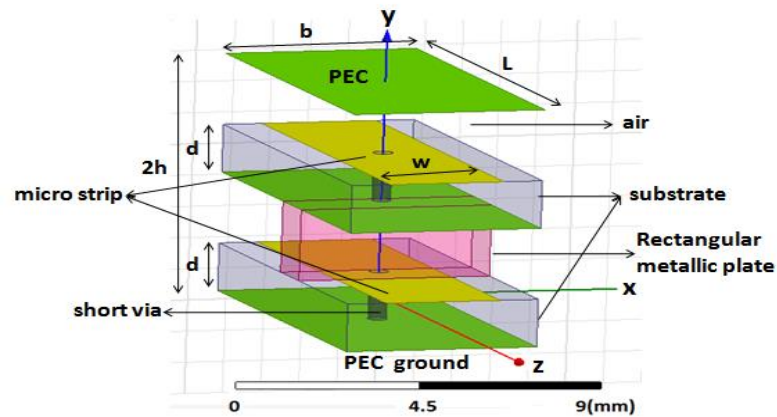

(a)

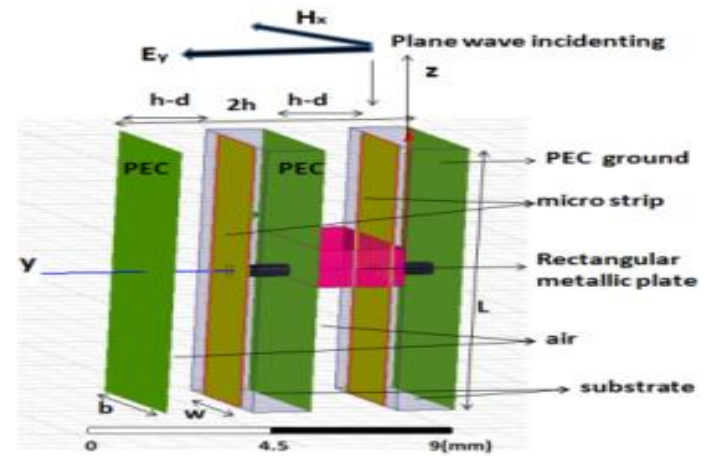

(b)

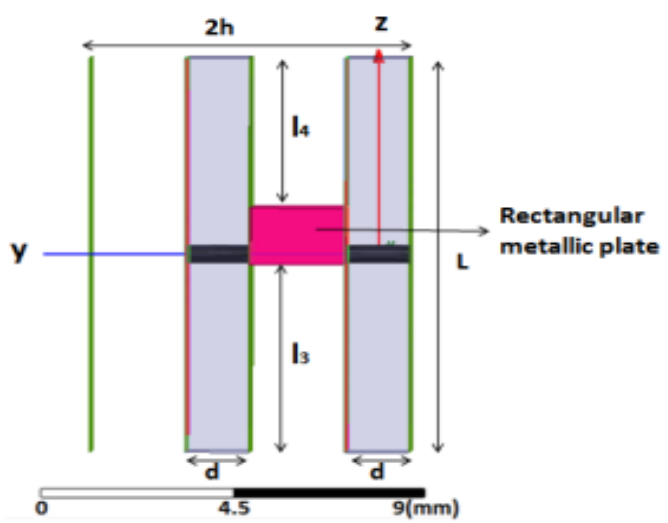

Figure 5. Three-dimensional view of a unit cell of (a) top view (b), (c) side view

\subsection{Equivalent circuit model}

The designed three dimensional band-pass FSS unit cell equivalent circuit model is shown in below Figure 6. $C_{a} \& C_{s}$ represent equivalent capacitors for the air to microstrip line discontinuity in the air and substrate regions of the designed unit cell respectively. $\mathrm{L}_{\mathrm{s}}$ is used for the equivalent inductor of short via. The air region with the rectangular metallic plate between the micro strip patch and ground is divided into two reflecting paths, they are represented by two shorted transmission lines $\left(Z_{a}, \Theta_{13}\right)$ and $\left(Z_{a}, \Theta_{14}\right)$. The other air region is represented by an open-circuited transmission line $\left(\mathrm{Z}_{\mathrm{a}}, \Theta_{\mathrm{a}}\right)$.Both the substrate regions of the unit cell are represented by two paths of same electrical length $\Theta_{\mathrm{s}} / 2$ and same characteristic impedance $Z_{\mathrm{s}}$.
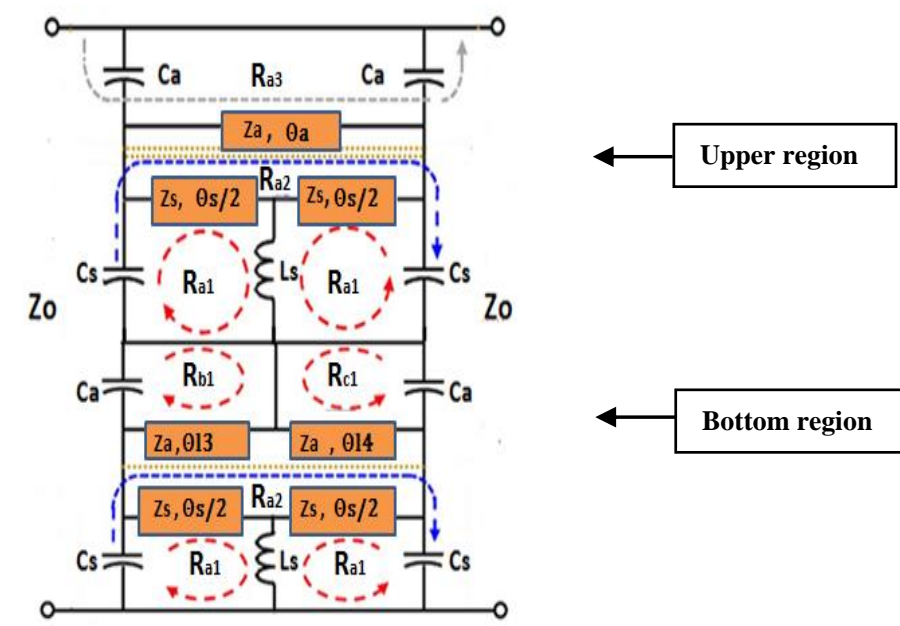

Figure 6. Equivalent circuit model of the unit cell for 3D band-pass FSS with five resonators and four transmission zeros 
The characteristic impedance for the guided region outside the unit cell with air is $\mathrm{Z}_{\mathrm{o}}$. There are total five resonators obtained as compared to the maximum four resonators obtained in the previous designs [14]. These resonators are obtained when a plane wave (TE polarization) incidents on the air to microstrip line discontinuity and the field propagates through different regions of the unit cell.

\subsection{Result and Discussion}

The Scattering parameters simulated results are shown in Figure 7. These results are used to analyse the band-pass characteristics of the designed 3D FSS structure.

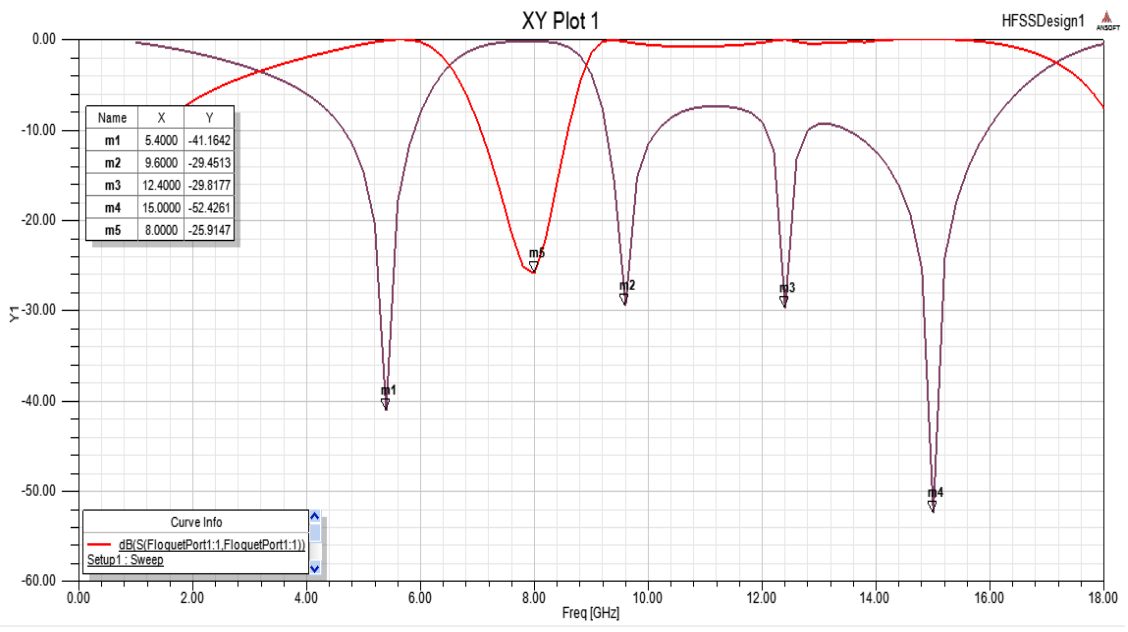

Figure 7. Sparameter results using HFSS software for the three dimensional band-pass

Figure 7 results consists of S11 (Reflection Coefficient) and S21 (Transmission Coefficient) parameters for the designed three dimensional FSS structure. The graph shows four transmission zeros at $5.4 \mathrm{GHz}, 9.6 \mathrm{GHz}, 12.4 \mathrm{GHz}$ and $15 \mathrm{GHz}$ frequencies. These transmission zeros consist of S21 (Transmission Coefficient) at $-41 \mathrm{~dB},-29.4 \mathrm{~dB}, 29.8 \mathrm{~dB}$ and $52 \mathrm{~dB}$. This fourth transmission zero in the operating frequency band provides wide out of band rejection [14]. The graph shows one transmission pole at frequency $8 \mathrm{GHz}$ with S11 (Reflection Coefficient) of $-25 \mathrm{~dB}$ which provides high selectivity. The Figure 8 shows in (a), (b), (c) and (d) parts, the simulated frequency responses for TE polarisation incident wave at various angles of incidence. It can be seen that S11 (Reflection Coefficient) and S21 (Transmission Coefficient) parameters at different resonance frequencies are decreasing with the increase of angle of incidence at 200, 400, and 600 respectively. The Figure 8(d) shows that frequency response is not having any transmission zero and pole of designed FSS. These types of 3D FSS can be used as pass band spatial filters allowing the desired band signals with reduced radar cross section of antenna design which is very important in stealth technology.

A 3D FSS is design and analysed with dual micro strip patch with substrate and rectangular metal plate with via hole shorted inside both the substrates. The wavelength of signals in the substrate path is lower than that in air $[14,17]$. The structure is analysed using equivalent circuit approach and designed in HFSS software.

\subsection{Deriving the $\mathrm{S}$ parameters for the 3D band-pass FSS}

The designed unit cell FSS of 3D band-pass in Figure 5(a) is obtained by combining two unit cells in Figure 3(a,b). The designed unit cell equivalent circuit is shown in Figure 6. We can observe four sections from this equivalent model, two air sections and two substrate sections. Each section can be represented by the transfer matrix which when cascaded would represent the transfer matrix of the entire unit cell of the designed 3D FSS structure. The Scattering parameters $S_{11}$ and $S_{21}$ are obtained by A, B, C, D parameters of the transfer matrix [14]. The transfer matrix of the different sections is shown through the block diagram as shown in Figure 9. Block 1 shows the transfer matrix for the air section with no metallic plate, Block 2 and Block 4 shows the transfer matrix for the substrate sections that are identical and Block 3 shows the transfer matrix for the air section having metallic plate. 


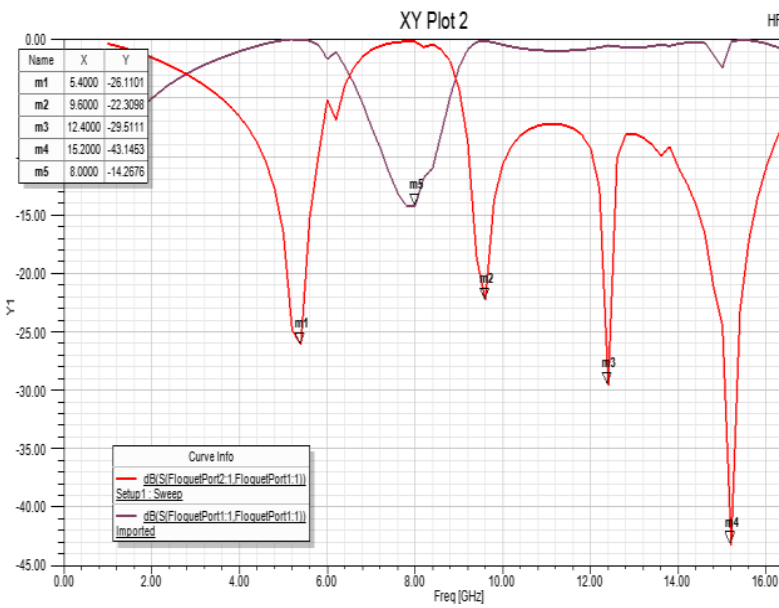

(a)

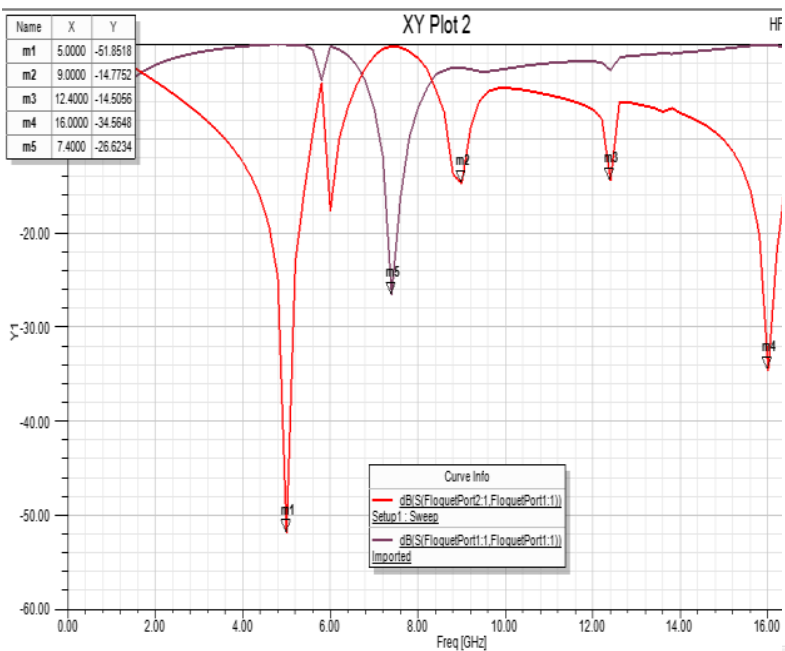

(c)

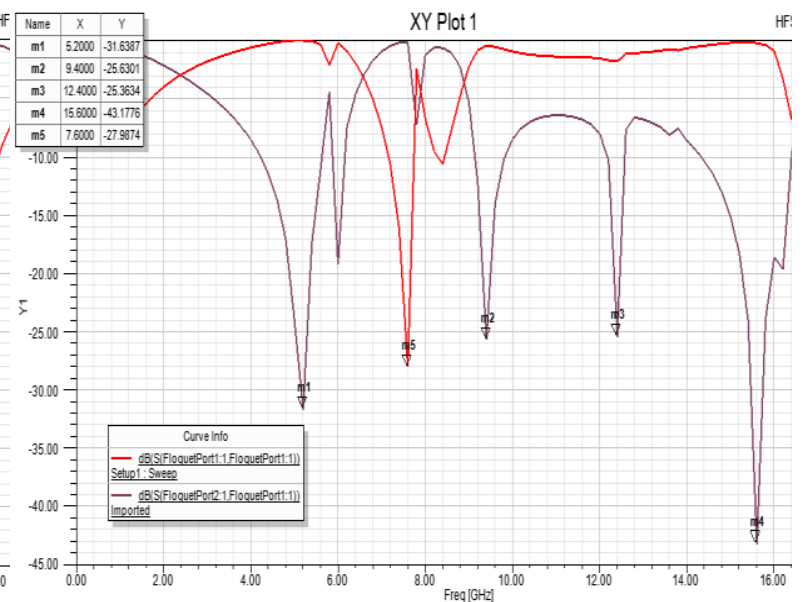

(b)

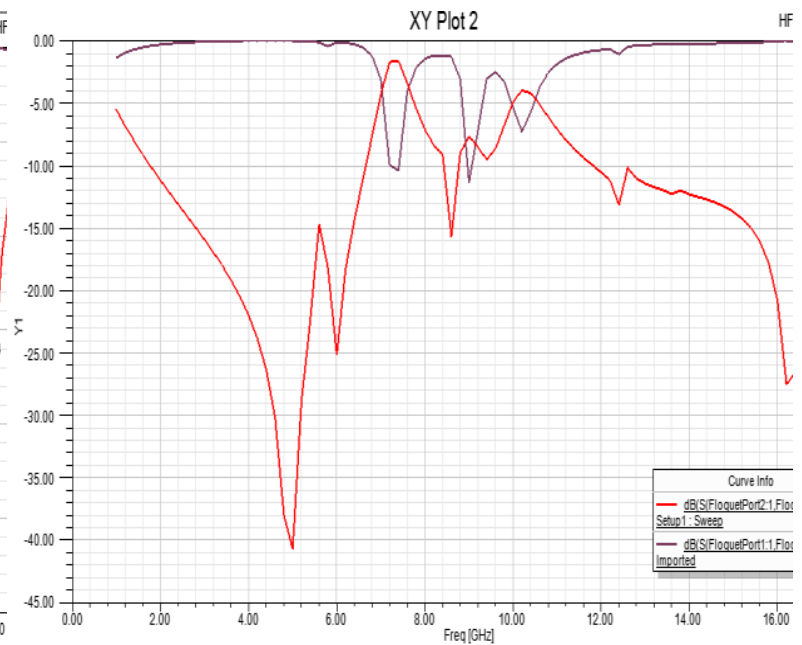

(d)

Figure 8. Simulated $\mathrm{S}$ parameters results for various angles of incidence of TE polarized plane wave. The angles of incidence are (a) 200 (b)400 (c)600 (d)800

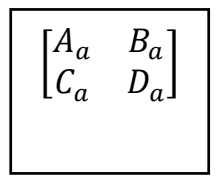

1

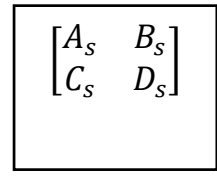

2

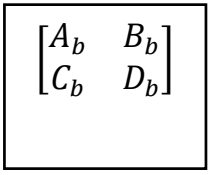

3

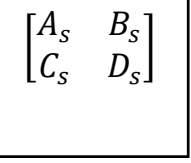

4

Figure 9. Block diagram having the transfer matrix of the four sections of the equivalent circuit model of the designed unit cell

These parameters A, B, C, D of the transfer matrix for the different sections depend onC $C_{a}, C_{s}, L_{s}, \Theta_{a}$, $\Theta_{\mathrm{s}}, \Theta_{13}, \Theta_{14}, Z_{\mathrm{a}}, \mathrm{Z}_{\mathrm{s}}$ and $\mathrm{Z}_{\mathrm{o}}$. From the matrix equations 1 and 7 as given in [14] the transfer matrix of each section can be obtained. The each section transfer matrix can be obtained by cascading the transfer matrix of each of its sub-section which is shown through a block diagram in Figure 10. 


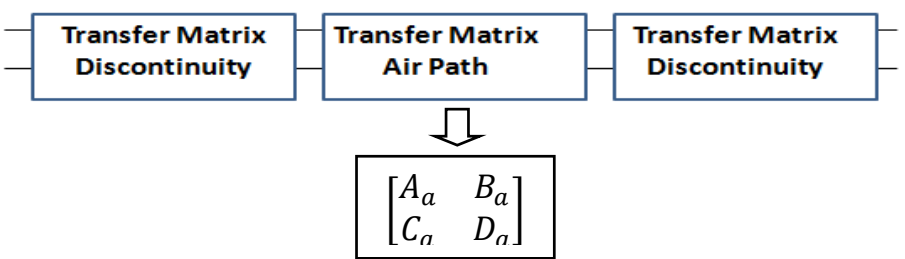

(a)

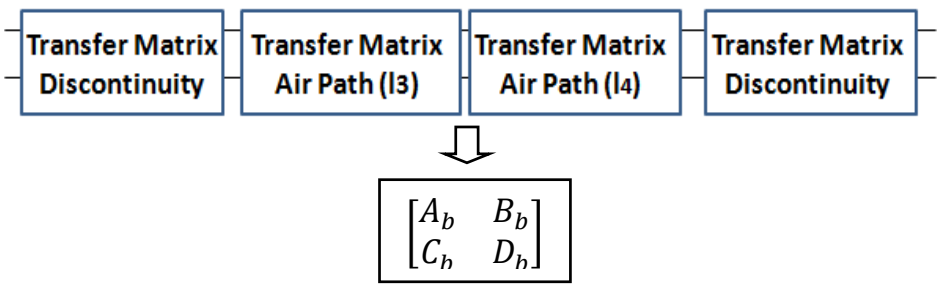

(b)

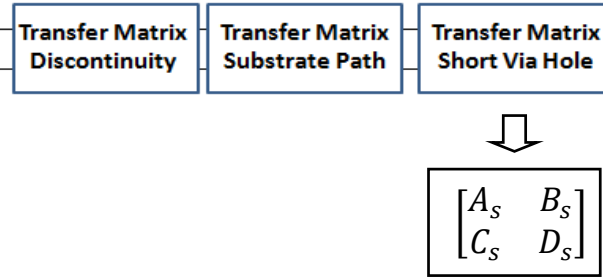

(c)

Figure 10. Block diagram showing the transfer matrix of the four sections and their sub-sections with the equivalent circuit model of the unit cell. (a) air section with no metallic plate, (b) air section with metallic plate, (c) two identical substrate sections

Transfer matrix of Block 1 and Block 2 in Figure 9 are cascaded together to obtain the Transfer matrix of the upper section of the unit cell and Transfer matrix of Block 3 and Block 4 are cascaded together to obtain the Transfer matrix of the bottom section of the designed unit cell in Figure 10. Thereafter the transfer matrix for the entire equivalent circuit model shown in Figure 6 for the designed unit cell can be obtained as shown in Figure 11, which is a cascade combination of the transfer matrix of the upper and bottom region of the equivalent circuit model.

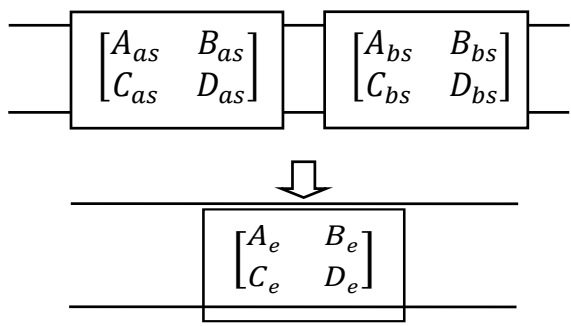

Figure 11. Block diagram having the transfer matrix of the upper region and bottom region of the equivalent circuit model of the designed unit cell. Their cascade combination gives the equivalent transfer matrix

The scattering parameters (reflection and transmission coefficient) for the entire equivalent circuit model are obtained as [14]:

$$
\mathrm{S}_{11}=\frac{\mathrm{A}_{\mathrm{e}}+{ }^{\mathrm{B} e} / \mathrm{z}_{0}-\mathrm{C}_{\mathrm{e}} \mathrm{Z}_{0}-\mathrm{D}_{\mathrm{e}}}{\mathrm{A}_{\mathrm{e}}+{ }^{\mathrm{B} e} / \mathrm{z}_{0}+\mathrm{C}_{\mathrm{e}} \mathrm{Z}_{0}+\mathrm{D}_{\mathrm{e}}}
$$


$\mathrm{S}_{21}=\frac{2}{\mathrm{~A}_{\mathrm{e}}+{ }^{\mathrm{B}} \mathrm{e} / \mathrm{z}_{0}+\mathrm{C}_{\mathrm{e}} \mathrm{Z}_{0}+\mathrm{D}_{\mathrm{e}}}$

The equivalent circuit parameters $\mathrm{C}_{\mathrm{a}}, \mathrm{C}_{\mathrm{s}}$ and $\mathrm{L}_{\mathrm{s}}$ are calculated by the physical dimensions of the designed unit cell structure as follows $[13,11]$ :

$$
\begin{aligned}
& \mathrm{C}_{\mathrm{a}} \approx \frac{\mathrm{w}}{\mathrm{b}} \frac{\mathrm{h}}{\omega(\mathrm{h}-\mathrm{d}) \mathrm{Z}_{0}} \tan \Delta \Theta \\
& \mathrm{C}_{\mathrm{s}} \approx \varepsilon_{\mathrm{r}} \frac{\mathrm{w}}{\mathrm{b}} \frac{\mathrm{h}}{\omega \mathrm{dz} \mathrm{Z}_{0}} \tan \Delta \Theta \\
& \mathrm{L}_{\mathrm{s}}=\frac{\mu_{0}}{2 \pi}\left[\mathrm{d} \ln 2 \frac{\mathrm{d}+\sqrt{\left(\frac{\mathrm{D}}{2}\right)^{2}+\mathrm{d}^{2}}}{\mathrm{D}}+1.5\left(\frac{\mathrm{D}}{2}-\sqrt{\left(\frac{\mathrm{D}}{2}\right)^{2}+\mathrm{d}^{2}}\right)\right]
\end{aligned}
$$

The characteristic impedance of the parallel plate waveguide $\mathrm{Z}_{0}$ represents the free space region outside the designed unit cell when carrying the TE polarized plane wave. The $\tan \Delta \Theta$ is evaluated from [14] as follows;

$$
\tan \Delta \Theta=\frac{2 \mathrm{~h}}{\lambda}\left(\frac{\mathrm{h}-\mathrm{d}}{\mathrm{h}} \ln \frac{\mathrm{h}}{\mathrm{h}-\mathrm{d}}+\frac{\mathrm{d}}{\mathrm{h}} \ln \frac{\mathrm{h}}{\mathrm{d}}\right)+\mathrm{S}_{1}\left(\frac{2 \mathrm{~h}}{\lambda} ; 0,0\right)-\mathrm{S}_{1}\left(\frac{2(\mathrm{~h}-\mathrm{d})}{\lambda} ; 0,0\right)-\mathrm{S}_{1}\left(\frac{2 \mathrm{~d}}{\lambda} ; 0,0\right)
$$

Where

$$
\mathrm{S}_{1}(\mathrm{x} ; 0,0)=\sum_{\mathrm{n}=1}^{\infty}\left(\sin ^{-1}\left(\frac{\mathrm{x}}{\mathrm{n}}\right)-\left(\frac{\mathrm{x}}{\mathrm{n}}\right)\right)
$$

The propagation constants of the air mode $\beta_{\mathrm{a}}$ and the substrate mode $\beta_{\mathrm{s}}$ are evaluated from [12], so the electrical lengths of air mode and substrate mode are realized as follows

$$
\begin{array}{r}
\text { Air mode: } \Theta_{\mathrm{a}}=\beta_{\mathrm{a}} \mathrm{l} \\
\Theta_{13}=\beta_{\mathrm{a}} \mathrm{l}_{3} \\
\Theta_{14}=\beta_{\mathrm{a}} \mathrm{l}_{4}
\end{array}
$$

Substrate mode: $\Theta_{s}=\beta_{s} l$

\subsection{Design Methodology}

The elementary design guidelines for designing the three dimensional band-pass FSS are as per [1], [16], [12].

a) The width $b$ and the height $2 * h$ of the unit cell should be smaller than the center frequency wavelength.

b) The microstrip length is calculated as

$$
\mathrm{L}=\frac{\lambda_{\mathrm{g}}}{2}=\frac{\lambda_{0}}{2 \sqrt{\varepsilon_{\mathrm{re}}}}
$$

$\lambda_{g}:$ Guided wavelength at the required center frequency of the FSS.

$\varepsilon_{r e}$ : Effective dielectric constant.

c) For the microstrip patch of thickness $t \rightarrow 0$ and $w / d \geq 1, \varepsilon_{r e}$ is evaluated [10] as follows

$$
\varepsilon_{\mathrm{re}}=\frac{\varepsilon_{\mathrm{r}}+1}{2}+\frac{\varepsilon_{\mathrm{r}}-1}{2}\left\{\left(1+12 \frac{\mathrm{d}}{\mathrm{w}}\right)^{-0.5}\right\}
$$

$\varepsilon_{r e}$ Is a function of frequency and its value lies between

$$
1<\varepsilon_{\mathrm{re}}<\varepsilon_{\mathrm{r}}
$$


As the frequency of operation increases $\varepsilon_{r e}=\varepsilon_{r}$.

The short via diameter $\mathrm{D}$ affects the first resonant frequency, which goes down to lower value as the diameter $\mathrm{D}$ decreases due to the dependency of the inductance Ls on diameter $\mathrm{D}$.

\section{CONCLUSION}

A 3D band-pass FSS structure has been designed by a $2 \mathrm{D}$ periodic array arrangement of dual surrounded microstrip lines. Each microstrip line in a unit cell is connected to its respective ground through short via hole. Two air regions are present above the microstrip lines with one having no metallic plate and the other having a rectangular metallic plate. Maximum four transmission zeros are obtained as compared to three transmission zeros obtained in [14]. Thus the designed three dimensional band-pass FSS exhibits high selectivity, elliptical frequency response, broad frequency band rejection and stable frequency result for different angles of incidence.

\section{REFERENCES}

[1] B.A.Munk, "Frequency Selective Surfaces: Theory and Design". New York, NY, USA: Wiley, 2000.

[2] S.N.Azemi,K. Ghorbani, "Frequency Selective Surfaces", Progress In Electromagnetics Research C,Vol.29,191$203,2012$.

[3] A.Mckay,B.Sanz-Izquierdo, "Evolution of Frequency Selective Surfaces", Forum for Electromagnetic Research Methods and Application Technologies(FERMAT).

[4] Tariq Rahim and Jiaodong Xu, "X-bandBand- pass Frequency Selective Surface for Radome Application", TELKOMNIKA Indonesian Journal of Elcerical Engineering, Vol. 16, No. 2. November 2015,pp,281-285.

[5] Umair Rafique, Syed Ahsan Ali, M.Tausif Afzal and Mudassar Abdin, "Bandstop Filter Design for GSM Shielding using Frequency Selective Surfaces", International Journal of Electrical and Computer Engineering, Vol.2.NO.6.December 2012pp.846- 850.

[6] K. Rashid and Zhongxiang Shen, "Three-dimensional FrequencySelective Surfaces", 978-1-4244-82238/10/\$26.0@2010 IEEE.

[7] Bo Li and Zhongxiang, "Three -Dimensional Band- Stop Frequency Selective Structures", 978-4673-21853/12/\$31.00@2012IEEE.

[8] Amir Khurrum Rashid, Zhongxiang Shen "An Elliptical Bandpass Frequency Selcetive Structure based on Microstrip Lines", IEEETransaction on Antenna and Propagation, Vol.60,No 10, October 2012.

[9] K. Rashid and Z. Shen, "Three-dimensional Monolithic Frequency Selective Structure with Dielectric Loading," Proceedings of Asia- pacific MicrowaveConference 2010.

[10] Abbas Abbaspour-Tamijani,Kamal Sarabandi "Antenna Arrays as a Class of Bandpass Frequency -Selective Surfaces", IEEE Transaction on Microwave Theory and Techniques, Vol. 52, no. 8, Aug. 2004.

[11] Marc E. Goldfarb and Robert A. Pucel, "Modeling Via Hole Grounds in Microstrip", IEEE Microwave and Guided Waveletters, Vol. 1, no. 6, June 1991.

[12] K. Rashid, Z. Shen, and S. Aditya, "Wideband microwave absorber based on a two-dimensional period array of microstrip lines," IEEE Transactions on Antennas and Propagation, Vol. 58, no.12, pp. 3913-3922, Dec. 2010.

[13] Ji- Fuh Liang, Xiao-Peng Liang, "Dual -Mode Dielectric or Air Filled Rectangular Waveguide Filters", IEEE Transactions on Microwave Theory and Techniques, Vol.42.No7,July 1994.

[14] Bo Li and Z. Shen, "Three-dimensional bandpass frequency- selective structures with multiple transmission zeros", IEEE Transaction on Microwave Theory and Techniques, Vol. 61, no. 10,Oct. 2013.

[15] BoLi and Zhongxiang Shen, "Three Dimensional Broadband Band Stop Frequency Selective Surface", 978-1-46735317-5/13/\$31.0@2013 IEEE.

[16] K. Rashid and Z. Shen, "A novel band-reject frequency Selectivesurface with pseudo-elliptic response", IEEE Transaction on Antennas Propagation, Vol. 58, no. 4, pp. 1220-1226, April. 2010.

[17] David M. Pozar, "Microwave Engineering", John Wiley \& Sons, Inc. 